Revista de Investigación Cientiffica y Tecnolo̊gica

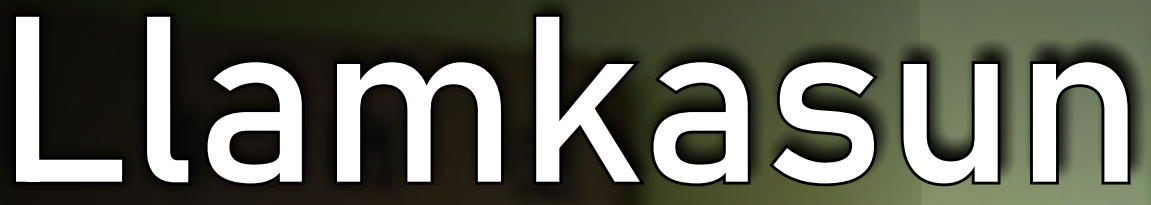

ISSN: 2709-2275

Trabajemos

Vol. 02 Num. 03 - Junio 2021

Incidencia de aborto en tiempos de COVID-19 en el Hospital de Lircay II-1, marzo 2020 a febrero 2021

Incidence of abortion in times of COVID-19 at Lircay II-1 Hospital, March 2020 to February 2021

(10.) Incidência de aborto em tempos de COVID-19 no Hospital Lircay II-1, Março de 2020 a Fevereiro de 2021

Edición Especial

COVID - 19

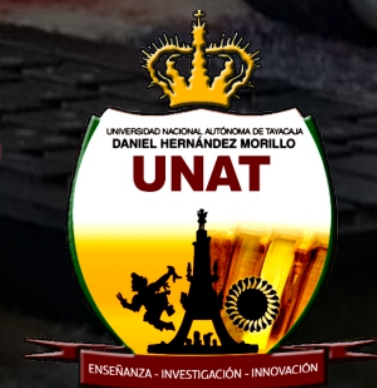

UNIVERSIDAD NACIONAL AUTÓNOMA DE TAYACAJA DANIEL HERNÁNDEZ MORILLO

VICEPRESIDENCIA DE INVESTIGACIÓN 


\title{
Incidencia de aborto en tiempos de COVID-19 en el Hospital de Lircay II-1, marzo 2020 a febrero 2021
}

\section{Incidence of abortion in times of COVID-19 at Lircay II-1 Hospital, March 2020 to February 2021}

\section{Incidência de aborto em tempos de COVID-19 no Hospital Lircay II-1, Março de 2020 a Fevereiro de 2021}

\author{
Richar Mallcco Quispe \\ Universidad Nacional de Huancavelica \\ Xiomara Alena Gamarra Taype ORCID \\ Universidad Nacional de Huancavelica \\ Jenny Mendoza Vilcahuaman ORCID \\ Universidad Nacional de Huancavelica
}

\section{RESUMEN}

El trabajo de investigación tuvo como objetivo determinar la incidencia del aborto en tiempos de COVID-19 en el hospital de Lircay II-1 de marzo 2020 - febrero 2021. El estudio fue de tipo observacional, retrospectivo, transversal y descriptivo; la muestra fue de 72 gestantes con diagnóstico de aborto y seleccionadas mediante los criterios de inclusión; se utilizó la técnica de análisis documental y el instrumento la ficha de recolección de datos. Los resultados muestran que la incidencia de aborto fue 4,8\% y de estos abortos el 29,2\% de mujeres fueron COVID-19 positivas; las características epidemiológicas encontradas fueron: la edad de 20 a 29 años, nivel de instrucción secundaria 55\%, estado civil conviviente 41,7\%, dentro de las características obstétricas, con una edad gestacional $<12$ semanas, son multigestas el 45,8\% ; la característica clínica predominante fue el sangrado vaginal con un70,8\%, la complicación más frecuente fue la anemia con un 78\% y más de la mitad de los abortos fue incompleto $75 \%$. En conclusión, la incidencia de aborto en tiempos de COVID - 19 en el hospital de Lircay II 1 en el periodo marzo 2020 a febrero 2021 fue baja.

Palabras clave: aborto, incidencia, características obstétricas, epidemiológicas.

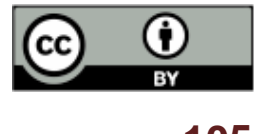




\begin{abstract}
The objective of the research work was to determine the incidence of abortion in times of COVID-19 in the Lircay II-1 hospital from March 2020 - February 2021. The study was observational, retrospective, cross-sectional and descriptive; the sample consisted of 72 pregnant women diagnosed with abortion and selected according to the inclusion criteria; the documentary analysis technique was used and the data collection form was used as the instrument. The results show that the incidence of abortion was $4.8 \%$ and of these abortions 29.2\% of women were COVID-19 positive; the epidemiological characteristics found were: age 20 to 29 years, high school education level 55\%, cohabiting marital status $41.7 \%$, within the obstetric characteristics, with a gestational age $<12$ weeks, are multigestational $45.8 \%$; the predominant clinical characteristic was vaginal bleeding with $70.8 \%$, the most frequent complication was anemia with $78 \%$ and more than half of the abortions were incomplete $75 \%$. In conclusion, the incidence of abortion in times of COVID - 19 in Lircay II - 1 hospital in the period March 2020 to February 2021 was low.
\end{abstract}

Keywords: abortion, incidence, obstetric, epidemiologic, characteristics.

\title{
RESUMO
}

O objectivo do trabalho de investigação era determinar a incidência do aborto durante a COVID-19 no hospital Lircay II-1 de Março de 2020 - Fevereiro de 2021. O estudo foi observacional, retrospectivo, transversal e descritivo; a amostra consistiu em 72 mulheres grávidas diagnosticadas com aborto e seleccionadas utilizando os critérios de inclusão; a técnica de análise documental foi utilizada e o formulário de recolha de dados foi utilizado como instrumento. Os resultados mostram que a incidência de abortos espontâneos foi de 4,8\% e que destes abortos 29,2\% das mulheres foram COVID-19 positivos; as características epidemiológicas encontradas foram idade 20-29 anos, nível secundário 55\%, estado civil em coabitação 41,7\%, dentro das características obstétricas, com uma idade gestacional <12 semanas, são multigestacionais $45,8 \%$; a característica clínica predominante foi hemorragia vaginal com 70,8\%, a complicação mais frequente foi anemia com 78\% e mais de metade dos abortos foram incompletos $75 \%$. Em conclusão, a incidência de aborto em tempos de COVID - 19 em Lircay II - 1 hospital no período de Março de 2020 a Fevereiro de 2021 foi baixa.

Palavras-chave: aborto, incidência, obstetrícia, epidemiológica, características. 


\section{INTRODUCCIÓN}

Se define como aborto a la detención espontánea o provocada del embarazo antes de las 22 semanas de gestación, que será contado desde el primer día de la última menstruación, con un peso menor o igual a $500 \mathrm{gr}$ (Guevara et al.. 2018).

La Organización Mundial de Salud menciona que cada año ocurre un aproximado de 74 millones de mujeres que tienen un embarazo no deseado y no planificado que constituyen la causa de 25 millones de abortos y 47000 muertes maternas al año; donde la mayoría de abortos (97\%) se producen en África, Asia y América Latina (Organización Mundial de la Salud, 2017).

El Instituto Guttmacher, en un estudio, nos menciona que entre los años de 2015 y 2019 ocurrieron aproximadamente 121 millones de embarazos no planificados, de las cuales un $61 \%$ terminó en aborto; es decir, que la incidencia de aborto a nivel mundial es aproximadamente 73 millones que sucedieron cada año; la tasa de aborto en países de ingresos bajos fue de $40 \%$, en países de ingresos medios $66 \%$ y en países de ingresos altos fue de 43\% (Institute Guttmarcher, 2020).
Con base del Instituto de Opinión Pública, el 19\% de las mujeres peruanas entre la edad de 18 a 49 años de edad refiere haberse realizado un aborto, estos datos no varían en las regiones; sin embargo, en el oriente se observa cifras más altas; esta práctica se lleva a cabo en todos los niveles socioeconómicos; el 58\% de las mujeres peruanas acudieron a un profesional de salud y el método más usado es la intervención quirúrgica (Pontificia Universidad Católica del Perú, 2018).

La interrupción del embarazo nos representa un problema de salud pública puesto que compromete a la salud reproductiva de las mujeres en edad fértil, desde el punto biológico, psicológico y social (Donis et al. 2010).

El aborto es un problema de salud pública que afecta a escala mundial principalmente a mujeres en edad reproductiva y que ocurren en todos los países sin importar el nivel de ingresos económicos, influyendo mayormente a países en vías de desarrollo mostrando una incidencia que surge cada vez más creciente y con una estadística más difícil de predecir, además provoca altos índices de complicaciones como hemorragias, lesiones en la cavidad uterina, sepsis e inclusive la mortalidad materna. 
En el Perú en la actualidad la realización de abortos es un tema ilegal, exceptos en casos de hallarse en peligro la vida de la mujer, o en casos donde el embarazo haya sido producto de una violación y la mujer tenga alguna enfermedad psiquiátrica (Díaz et al., 2019).

Un estudio realizado en Lima en el hospital nacional Sergio Bernales el año 2018, halló que la incidencia de las pacientes que presentaron aborto en mayor frecuencia fue entre las edades de 20 a más años (73,3\%), el grado de instrucción de secundaria $(79,2 \%)$, en la ocupación eran amas de casa $(78,3 \%)$, el estado civil fue conviviente (71,7\%), en la edad gestacional que más frecuentemente encontrada osciló entre 11 a 15 semanas de gestación(56,7\%), las pacientes que presentaban antecedente de aborto representó el $(31,7 \%)$ y en antecedentes de gestaciones presentó nuliparidad el $61,5 \%$ de las pacientes (Huertas, 2018).

En el servicio de Gineco-Obstetricia del hospital de Lircay ingresan frecuentemente pacientes con este diagnóstico por lo que el porcentaje de abortos se desconoce actualmente, ante la cual se dio la inquietud de poder dar a identificar las características de las pacientes que ingresan por aborto para así evitar complicaciones en el futuro y se mejore la calidad de salud en nuestro medio, de esta manera poder enfrentar el problema de salud como el aborto en tiempos de COVID-19.

El aborto es un problema y seguirá siéndolo, su práctica no debe de poner en riesgo la salud de las mujeres; es por ello, que existen diversos tratamientos para atender los casos de aborto; sin embargo, este va a depender según la clasificación y gravedad.

Esta investigación tuvo el propósito de determinar la incidencia de aborto y describir las características epidemiológicas, obstétricas, clínicas y complicaciones del aborto, de mujeres de los Andes del Perú, atendidas en tiempos de COVID-19 en el hospital de Lircay II-1 de Huancavelica.

\section{METODOLOGÍA}

La investigación fue de tipo observacional, retrospectivo, transversal y descriptivo. Según la clasificación de Centi (2006), el nivel de investigación es descriptivo: porque está dirigido a describir cual es el problema y como se está manifestando; es por ello, que se busca determinar cuál es la incidencia de aborto en tiempos de covid-19 en el hospital Lircay II -1 de marzo 2020 a febrero 2021, así también se buscó especificar las propiedades importantes del objeto de investigación, seleccionando una 
serie de aspectos para luego medirlas independientemente, para así describir lo que se investiga.

El estudio se realizó mediante un diseño no experimental y descriptivo simple, porque no se manipulan variables y se basa en realidades del hecho (Centi, 2006).

Dónde:

$\mathrm{M} \rightarrow \mathrm{O}$

M: Mujeres con diagnóstico de aborto

O: Características de las mujeres con diagnóstico de aborto.

La población de estudio de gestantes con diagnóstico de aborto, según los datos registrados en el establecimiento de salud, fueron un total de 72 mujeres.

El tipo de muestra fue censal, seleccionadas de acuerdo a los criterios de inclusión del estudio de investigación.

En los criterios de inclusión, se consideró; mujer con diagnóstico de aborto, pacientes atendidas en el servicio de ginecoobstetricia con y sin atención prenatal. Los criterios de exclusión fueron: Mujeres con diagnóstico de aborto cuyas historias clínicas estaban incompletas, ilegible y con enmendaduras.

La técnica de investigación utilizada fue la encuesta, y el instrumento fue el cuestionario (Bravo y Valenzuela 2019). Para la recolección de datos, se solicitó la autorización al director del Hospital de
Lircay de Huancavelica, se coordinó con la jefa de servicio de obstetricia para la selección de las historias clínicas de los sujetos de investigación, se recogió los datos usando el instrumento correspondientes.

Para el análisis y procesamiento de datos, se realizó la tabulación de la información en la hoja de cálculo Microsoft Excel, y para el análisis estadístico se empleó el programa estadístico SPSS versión 23.0, con el que se realizó un análisis de acuerdo a la naturaleza del estudio.

\section{RESULTADOS}

Los resultados nos muestran que la incidencia del aborto en mujeres atendidas en el hospital de Lircay en tiempos de COVID-19 fue de 4,8\%. De ellas $21(29,2 \%)$ de mujeres fueron COVID-19 positivas.

Las características epidemiológicas de las mujeres con diagnóstico de aborto en tiempo de covid-19 en el hospital Lircay ii1 marzo 2020 - febrero 2021, nos muestran que de las 72 mujeres que fueron atendidas por aborto, tenían de 10 a 19 años 5 (6,9\%), 20 a 29 años $50(69,4 \%)$ y 30 a 50 años 17 $(23,7 \%)$; instrucción primaria $16(22,2 \%)$, secundaria $40(55,6 \%)$, superior técnico 7 $(9,7 \%)$ y superior universitario 9 (12,5\%); 
estado civil conviviente 30 (41,7\%), solteras $23(31,9 \%)$, casadas $25,0 \%$ (18) y divorciadas $1,4 \%$ (1).

Las características obstétricas de las mujeres con diagnóstico de aborto en tiempo de covid-19 en el hospital Lircay ii1 marzo 2020 - febrero 2021, nos muestran que de las 72 mujeres que fueron atendidas por aborto, tienen de 12 a menos semanas de embarazo 79,2\% (57) y de 13 a 22 semanas 20,8\% (15); sin atención prenatal (APN) $61,1 \%$ (44) y con APN 38,9\% (28); son primigestas $22,2 \%$ (16), según digestas $31,9 \%$ (23) y multigestas $45,8 \%$ (33); con cero hijos 31,9\% (23), un hijo 38,9\% (28), dos hijos $12,5 \%$ (9) y tres a más hijos 16,7\% (12); sin antecedente de aborto 56,9\% (41) y con antecedente de aborto 43,1\% (31); no usan método anticonceptivo (MAC) 86,1\% (62) y usan MAC 13,9\% (10).

Las características clínicas de aborto en las mujeres atendidas en tiempo de covid-19 en el hospital Lircay ii-1 marzo 2020 - febrero 2021, se observa que, de las 72 mujeres, tuvieron sangrado vaginal $70,8 \%$ (51), dolor hipogástrico $15,3 \%$ (11), malestar general $12,5 \%$ (9) y fiebre $1,4 \%$ (1).

Tipos de aborto en las mujeres atendidas en tiempo de covid-19 en el hospital Lircay ii1 marzo 2020 - febrero 2021, se observa que de 72 mujeres que fueron atendidas por aborto, tuvieron aborto incompleto $75,0 \%$
(54), completo $15,3 \%$ (11), retenido $5,6 \%$ (4) y frustro $4,1 \%$ (3).

Sobre el manejo del aborto en las mujeres atendidas en tiempo de covid-19 en el hospital Lircay ii-1 marzo 2020 - febrero 2021, se observó de un total de 100,0\% (72) mujeres que fueron atendidas por aborto, tuvieron legrado uterino $75,0 \%$ (54) y manejo farmacológico 25,0\% (18).

De las complicaciones de las mujeres con diagnóstico de aborto atendidas en tiempo de covid-19 en el hospital Lircay ii-1 marzo 2020 - febrero 2021, se observó que del $100,0 \%$ (72) mujeres que fueron atendidas por aborto, el $77,8 \%$ (56) gestantes presentaron complicaciones. De estas, presentaron anemia el 78,6\% (44); hemorragias $14,3 \%$ (8) y sepsis el 7,1\% (4).

\section{DISCUSIÓN}

La incidencia del aborto en mujeres atendidas en el hospital de Lircay en tiempos de COVID-19 fue 4,8\%; de ellas el 29,2\% fueron COVID-19 positivas. Resultado que difiere con Pincay y Villacis (2018) quienes en su investigación hallaron mayor incidencia de abortos en la población estudiada. Esto se debe a que los estudios se realizaron en contextos geográficos diferentes. El aborto pone en riesgo la vida de la mujer, por ello debe prevenirse con 
actividades de promoción de la salud en las mujeres en edad fértil (MEF).

Las características epidemiológicas frecuentes son: 20 a 29 años 69,4\%, 30 a 50 años 23,7\%; instrucción primaria 22,2\%, secundaria $55,6 \%$; convivientes $41,7 \%$ y solteras 31,9\%. Datos que coinciden con el estudio realizado por Mogrovejo (2018); quien encontró que el aborto se da en mujeres de 21 a 30 años el 64,9\%; el grado de instrucción secundaria en un 77,37\% y el estado civil conviviente con un $51,82 \%$ de su población estudiada. Esto nos indica que el nivel de instrucción secundaria, la edad joven y el estado civil conviviente pueden ser factores de riesgo para tener un aborto, por lo que sería interesante hacer un estudio de asociación entre el aborto y estas variables.

Las características obstétricas frecuentes: tienen de 12 a menos semanas de embarazo 79,2\%, 13 a 22 semanas 20,8\%; sin APN 61,1\%, con APN 38,9\%; primigestas $22,2 \%$, según digestas $31,9 \%$ y multigestas $45,8 \%$; sin hijos $31,9 \%$, un hijo $38,9 \%$, dos a más hijos 29,2\%; sin antecedente de aborto $56,9 \%$, con antecedente de aborto 43,1\%; no MAC $86,1 \%$ y usan MAC 13,9\%. En este estudio los abortos sucedieron de 12 a menos semanas de embarazo $79,2 \%$, lo que coincide con el estudio de Rivero (2017) en su estudio la semana gestacional del aborto más frecuente fue en menores de 12 semanas con un 79\%; así mismo con el estudio de Mogrovejo (2018) también hallo abortos en menos de 12 semanas en un $63,76 \%$ de gestantes. Quedando claro que los abortos en su mayoría suceden durante el primer trimestre de gestación, por lo cual se necesita que las APN sean más tempranas. En el presente estudio los abortos fueron más frecuentes en multigestas con un $45,8 \%$, resultado que concuerda con el estudio de Guerrero (2018) el cual obtuvo que el $51,4 \%$ de mujeres con aborto eran multigestas; con ello se pueda formar que los abortos suceden más en mujeres multigestas. En nuestro estudio respecto a los antecedentes de aborto, el 56,9\% de las mujeres no tuvieron antecedentes de aborto y el $43,1 \%$ si; resultado que concuerda con lo hallado por Mogrovejo (2018) en su estudio registró en un 52\% abortos en mujeres que no presentaron antecedentes. Esto nos permite ver que los abortos se dan en multigestas sin antecedentes, lo que hace necesario trabajar la promoción de MAC en MEF.

Las características clínicas del aborto frecuentes son: sangrado vaginal $70,8 \%$, dolor hipogástrico 15,3\%, malestar general $12,5 \%$ y fiebre $1,4 \%$, el resultado del sangrado vaginal, difiere con el estudio de 
Cenzano (2019), en su estudio encontró que el $100 \%$ de abortos presento sangrado vaginal. Algunos abortos espontáneos no dan sangrado vaginal abundante $\mathrm{y}$ se confunden con un ciclo menstrual, esto pudo suceder en el 29,2\% de mujeres de nuestro estudio.

Esta investigación encontró tipos de aborto: incompleto en el 75\%; completo 15,3\%; retenido $5,6 \%$; y frustro $4,1 \%$; resultado semejante a lo hallado por Guerrero (Guerrero, 2018), quien observó que el tipo clínico de aborto con mayor frecuencia es el incompleto con un 85,7\%. Los abortos incompletos son los más frecuentes y los que pueden producir más complicaciones en la salud de la mujer, siendo importante el manejo medico temprano de estos casos.

Esta investigación respecto al manejo del aborto encontró que; $54 \%$ necesito legrado uterino y el $25 \%$ manejo farmacológico; coincide con el estudio de Moreira (2018) encontró en un $51 \%$ a las mujeres que pasaron por un legrado uterino. Los abortos que se atienden en un establecimiento de salud por lo general terminan con un legrado para evitar complicaciones.

La complicación clínica del aborto más frecuente encontrado en nuestro estudio fue la anemia en un $78,6 \%$, seguido de hemorragia en un $8 \%$ y sepsis con $4 \%$; lo que no concuerda con el estudio de Pincay y Villancis (2018), quienes obtuvieron como complicación a la anemia en un $21 \%$. La anemia es una complicación frecuente, por lo que medir la hemoglobina pos aborto es una medida que se recomienda en todos los establecimientos e salud, lo que permitirá que la mujer pos aborto tenga una evolución favorable.

\section{CONCLUSIONES}

La incidencia de abortos en tiempos de COVID-19 en el hospital de Lircay II-1 de Huancavelica fue de 4,8\%, incidencia baja a pesar del contexto de la COVID- 19. Esto puede deberse a que los sujetos de estudio pertenecen a una población Alto Andina de la región central del Perú, donde la sociedad es más conservadora y reservada.

Las características epidemiológicas principales de las gestantes con diagnóstico de aborto, fueron edad joven de 20 a 29 años, con nivel de instrucción secundaria y estado civil conviviente.

Las características obstétricas frecuentes de las gestantes con diagnóstico de aborto fueron; los abortos sucedieron en la edad gestacional menor igual a 12 semanas, se dieron en las multigestas, en mujeres que no tuvieron antecedentes de aborto $\mathrm{y}$ en mujeres que no usaron anticonceptivos. 
Las características clínicas halladas en la mayoría de las mujeres con aborto fue el sangrado vaginal, y las menos frecuentes fueron; dolor en hipogastrio, malestar general y fiebre.

La principal complicación identificada en las gestantes con aborto fue la anemia, y las menos frecuentes fueron; la hemorragia y sepsis.

El manejo de las gestantes con diagnóstico de aborto fue en mayoría legrado uterino y solo la cuarta parte manejo farmacológico.

\section{REFERENCIAS BIBLIOGRÁFICAS}

Bravo, T. y Valenzuela, S. (2019). Desarrollo de instrumentos de evaluación: cuestionarios. Centro UC. https://www.inee.edu.mx/wpcontent/uploads/2019/08/P2A355.pd f

Centty, D. B. (2006). Manual metodológico para el investigador científico. Perú: $B$ Universidad Nacional de San Agustín de Arequipa, 30. https://scholar.google.com/scholar?clu ster $=17964300419841259345 \& \mathrm{hl}=\mathrm{en}$ \&oi=scholarr

Cenzano, W. V. (2019). Características sociodemográficas y clínicas de los abortos en mujeres del Hospital
Regional Docente Materno Infantil El Carmen, 2018. (Tesis de Especialización, Universidad Nacional de Huancavelica) https://repositorio.unh.edu.pe/bitstre am/handle/UNH/2639/TESIS-SEGESP-OBSTETRICIA-2019PAUCARCHUCO\%20CENZANO. pdf?sequence $=1 \&$ is Allowed $=y$

Diaz, J. J., Olorteguí, W. y Salazar, L. V. (2019) Periodo intergenésico corto como factor de riesgo para aborto recurrente en el primer trimestre de embarazo: estudio de casos y controles". Hampi Runa, 19(1). http://journal.upao.edu.pe/HAMPIR UNA/article/view/1456

Donis, N. I., Batista, I. D., \& Manrique, A. J. (2010). Aborto en la adolescencia un problema de salud. Revista Cubana de Obstetricia y Ginecología $36 \quad$ (3) 409-421. http://scielo.sld.cu/scielo.php?script $=$ sci_arttext\&pid=S0138600X2010000300011\&lng=es.

Guerrero, C. I. (2018) Características clínico epidemiológicas de las pacientes con diagnóstico de aborto en el hospital de apoyo nuestra señora de las Mercedes-Paita durante el período julio a diciembre 2017. 
(Tesis de titulación, Universidad. Nacional de Piura, Piura). https://repositorio.unp.edu.pe/handle /UNP/1184

Guevara, E. Sánchez, A., Luna A. M., Ayala, F. D., Mascaro, P.A., Carranza, C. A. y Meza, L. (2018) Guías de práctica clínica y de procedimientos en obstetricia y perinatología. Instituto Nacional Materno Perinatal, Lima. Perú (2da. Ed). Lima, Perú: Gráfica Delvi S.R.L.

Huertas, M. (2018). Características generales y obstétricas de pacientes con aborto espontaneo en el servicio de gineco-obstetricia del hospital de Ventanilla - Callao. (Tesis de titulación, Universidad San Martin de Porres, Lima, Perú). https://repositorio.usmp.edu.pe/hand le/20.500.12727/5535

Institute Guttmarcher (2020). Embarazo no planeado y aborto a nivel mundial.

https://www.guttmacher.org/sites/de fault/files/factsheet/fs-aww-es.pdf

Mogrovejo, M. S. (2018) Características epidemiológicas de pacientes con aborto espontáneo en el servicio de gineco-obstetricia del hospital nacional Sergio E. Bernales durante el periodo de enero-diciembre 2017. (Tesis de Titulación, Universidad Privada San Juan Bautista, Lima, Perú).

https://renati.sunedu.gob.pe/handle/s unedu/1674988

Moreira, V. N. (2018). Perfil epidemiológico, clínica y complicaciones de aborto en mujeres de 15 a 25 años, hospital Liborio Panchana. (Tesis de Titulación, Universidad de Guayaquil. Guayaquil, Ecuador). https://renati.sunedu.gob.pe/handle/s unedu/1674988

Organización Mundial de la Salud [OMS]. (2017). En todo el mundo se producen aproximadamente 25 millones de abortos peligrosos al año.

https://www.who.int/es/news/item/2 8-09-2017-worldwide-an-estimated25-million-unsafe-abortions-occureach-year

Pincay, D. M. y Villacis, M. V. (2018). Incidencia de aborto en mujeres entre 20 a 42 años de un Hospital De Especialidades De La Ciudad De Guayaquil. (Tesis de Titulación, Universidad Católica de Santiago de 
Guayaquil, Ecuador).

http://repositorio.ucsg.edu.ec/handle

/3317/10129

Pontificia Universidad Católica del Perú (2018). El aborto en cifras: Encuesta

a mujeres en el Perú. https://promsex.org/publicaciones/el -aborto-en-cifras-encuesta-amujeres-en-el-peru/

Rivero, N. M. (2017). Frecuencia y factores de riesgo de aborto en mujeres de 20 a 40 años en el hospital Mariana de Jesús durante el periodo de enero y febrero del 2017. (Tesis de titulación, Universidad Católica de Santiago de Guayaquil, Guayaquil, Ecuador). http://repositorio.ucsg.edu.ec/handle /3317/8043

\section{Contacto}

Richar Mallcco Quispe

2017321014@unh.edu.pe 